\section{P2.094 THE CONTRIBUTION OF MACROLIDE RESISTANCE MUTATIONS TO FAILURE OF AZITHROMYCIN TREATMENT IN MYCOPLASMA GENITALIUM INFECTION}

\author{
doi:10.1136/sextrans-2013-051184.0358
}

${ }^{1,2} \mathbf{M}$ Bissessor, ${ }^{1,2} \mathrm{C}$ K Fairley, ${ }^{1,2} \mathrm{M}$ Y Chen, ${ }^{3} \mathrm{~S} N$ Tabrizi, ${ }^{4} \mathrm{~J}$ Hocking, ${ }^{1,2,5} \mathrm{C}$ S Bradshaw. 'Melbourne Sexual Health Centre, Melbourne, Australia; 'Melbourne School of Population Health, University of Melbourne, Melbourne, Australia; ${ }^{3}$ Department of Microbiology Royal Women's Hospital, Melbourne, Australia; ${ }^{4}$ Centre for Women's Health, Gender and Society, University of Melbourne, Melbourne, Australia; ${ }^{5}$ Department of Epidemiology and Preventive Medicine, Monash University, Melbourne, Australia

Background Current recommended treatment for Mycoplasma genitalium $(\mathrm{Mg})$ is azithromycin. Macrolide resistance mutations (MRM), predominantly on the 23SrRNA gene of $\mathrm{Mg}$, have been found to be associated with failure of azithromycin. We aimed to determine the efficacy of $1 \mathrm{~g}$-azithromycin in a prospective cohort of Mg-infected STI clinic attendees, and to determine the contribution of MRM to treatment failure.

Method We commenced an observational study in July 2012 in which symptomatic patients diagnosed with Mg by PCR at Melbourne Sexual Health Centre are retested for Mg 14 and 28 days following treatment with $1 \mathrm{~g}$-azithromycin. Testing for MRM using high-resolution melt analysis (HRM) is conducted on day 0 and on positive samples at days 14 and 28. Participants are managed on the basis of clinical symptoms and not detection of MRM. Study will complete, May 2013.

Results 105 participants have been recruited; 89 have completed all study requirements. 48/89 (54\%; 95\% CIs 44-64\%) participants were Mg PCR negative at day 28. 41/89 (46\%; 95\% CIs 36-56\%) did not respond to $1 \mathrm{~g}$ azithromycin: 11/41 (27\%) had a persistently positive Mg PCR on day 28 without reported risk of re-exposure (presumptive failures) and 30 of 41 (73\%) had persistent symptoms of MG prior to day 28 and required interim treatment with moxifloxacin (probable failures). Of the 41 failures, 40 (98\%) had MRM detected: 30 (75\%) at baseline and $10(25 \%)$ at day 14 only. Of the 48 azithromycin-responders 4(8\%) had MRM detected at baseline.

Conclusion The azithromycin cure rate for $\mathrm{Mg}$ in this clinic cohort was only $54 \%$. MRM were detected in virtually all cases of azithromycin-failure, and were uncommon in azithromycin-responders. The majority of MRM were detected prior to treatment. These findings have implications for the use of macrolides as current recommended treatment for M.genitalium, and highlight the need for evaluation of alternative treatment approaches.

\section{P2.095 ORIGINS OF REPEAT INFECTIONS WITH MYCOPLASMA GENITALIUM (MG) AMONG HETEROSEXUAL MEN IN TWO SOUTHERN U.S. CITIES}

doi:10.1136/sextrans-2013-051184.0359

'P Kissinger, 'S White, ' $\mathrm{N}$ Schmidt, ${ }^{2} \mathrm{~S} N$ Taylor, ${ }^{3} \mathrm{~L}$ Mena, ${ }^{2} \mathrm{R}$ Lillis, 'S A Some, K Defayette, ${ }^{12}$ D H Martin. 'Tulane University SPHTM, New Orleans, LA, United States, ${ }^{2}$ Louisiana State University Health Sciences Center, New Orleans, LA, United States, ${ }^{3}$ University of Mississippi - Department of Medicine, Jackson, MS, United States

Background The purpose of this study was to examine the origins of repeat infections with $\mathrm{Mg}$ among men. High repeat infection rates have been consistently reported and treatment failure secondary to macrolide resistance is thought to be the primary cause. This study adds to the growing literature by describing the possible origins of repeat $\mathrm{Mg}$ infections in among men, primarily African American, attending two public STD clinics in southern cities in the U.S.

Methods Men diagnosed with NGU at an STD clinic in New Orleans, Louisiana, and Jackson, Mississippi, were tested for $\mathrm{Mg}$ using the GenProbe research-use-only assay $\mathrm{Mg}+$ men underwent a 4-10 week test of cure visit (TOC) following treatment with $1 \mathrm{~g}$ azithromycin. Detailed sexual behaviour data were collected at baseline and follow-up via ACASI and genotyping was performed. Results Of 205 men with MG, 135 returned for TOC visit and of those, $34.3 \%$ were positive. Of the 46 who were positive at TOC, $19.6 \%$ reported sexual re-exposure to a baseline partner, $6.5 \%$ reported sexual exposure to a new partner, $6.5 \%$ reported sexual exposure to both, and $67.4 \%$ denied sexual re-exposure. Men who re-tested positive for $\mathrm{Mg}$ at TOC $(\mathrm{n}=46)$ were no more likely that those who tested negative $(n=88)$ to have had sexual exposure to a baseline partner $(31.8 \%$ vs. $26.1 \%, \mathrm{P}=0.55)$ or exposure to a new partner $(21.4 \%$ vs. $11.3 \%, \mathrm{P}=0.22)$. Genotyping on baseline/TOC positive pairs is being performed and will be used in conjunction with behavioural data to more precisely estimate the treatment failure rate.

Conclusion The TOC repeat infection rate among men with $\mathrm{Mg}$ is high. Our data are consistent with the published literature corroborating that repeat infections in men treated with azithromycin is usually due to treatment failure rather than re-infection. Research is needed to optimise treatment of $\mathrm{Mg}$ infections in men.

\section{P2.096 EVALUATION OF GONOCOCCAL RESISTANCE IN AUSTRIA}

doi:10.1136/sextrans-2013-051184.0360

'A Stary, ${ }^{1} \mathrm{M}$ Haller, ${ }^{1} \mathrm{C}$ Heller-Vitouch, ${ }^{1} \mathrm{~N}$ Hartl, ${ }^{2} \mathrm{E}$ Vinzelj-Horvath, ${ }^{3} \mathrm{~A}$ Geusau, ${ }^{4} \mathrm{~K}$ Rappersberger, ${ }^{5} \mathrm{P}$ Komericki, ${ }^{6} \mathrm{~J}$ Auböck, ${ }^{7} \mathrm{R}$ Hoepfl. ' Outpatient's Centre for Diagnosis of Infectious Venereodermatological Diseases, Vienna, Austria; ${ }^{2}$ Outpatient's Clinic for Sexual Transmitted Diseases, Vienna, Austria; ${ }^{3}$ Department of Dermatology, Division of Immunology, Allergy and Infectious Diseases (DIAID), Medical University of Vienna, Vienna, Austria; ${ }^{4}$ Department of Dermatology and Venereology, Community Hospital Krankenanstalt Rudolfstiftung, Vienna, Austria; ${ }^{5}$ Department of Dermatology and Venereology, Medical University of Graz, Graz, Austria; ${ }^{6}$ Department of Dermatology and Venereology, General Hospital, Linz, Austria; 'Department of Dermatology and Venereology, Medical University of Innsbruck, Innsbruck, Austria

Background The Austrian Society for Sexually Transmitted Diseases started a surveillance project in 2010 to evaluate the gonococcal resistance in Austria.

Methods In 2010 and 2011 a national network of 39 centres was established to collect 1569 gonococcal isolates and the anamnestic data of the patients. Gonococcal culture plates were sent from the participating laboratories to the Outpatient's Center in Vienna, where gonococcal resistance testing was performed by disc diffusion test, agardilution breakpoint technique, agardilution, and Etest. The following antibiotics were tested: cefixime, ceftriaxone, penicillin, ciprofloxacin, azithromycin, tetracycline, spectinomycin, and gentamicin. Results were interpreted according to CLSI and EUCAST guidelines.

Results In Vienna, 1456 isolates were collected, whereas 111 strains were sent from the federal states. Of all collected isolates $87 \%$ were genital, $5 \%$ pharyngeal, and $7 \%$ rectal isolates, respectively. Gonococci were collected more often from men $(56 \%)$ than from women, $10 \%$ of men reported homosexual contacts. A concurrent infection with Chlamydia trachomatis was observed in $15 \%$ of all patients.

While 2010 all isolates displayed susceptibility to third generation cephalosporines, in 20117 gonococcal strains were resistant to cefixime (MIC $>0.125 \mu \mathrm{g} / \mathrm{ml}$ ) but still susceptible to ceftriaxone (MIC $\leq 0.125 \mu \mathrm{g} / \mathrm{ml}$ ). Furthermore, an increase of MIC values for cefixime as well as for ceftriaxone was observed in 2011. Resistance to azithromycin increased from $1 \%$ in 2010 to $1.5 \%$ in 2011. Resistance to quinolones was detected in $58 \%$ in 2010 rising to more than $60 \%$ in 2011, respectively.

Conclusion Third generation cephalosporines still represent the most appropriate drug for gonococcal therapy. As Azithromycin resistance is low in Austria it is suitable for alternative therapy especially in case of coinfection with C. trachomatis. Due to the increasing MIC values for cephalosporines and the rising rates for 